\title{
Presentation of Dark Matter Candidates
}

\author{
Koshun Suto ${ }^{1}$ \\ ${ }^{1}$ Chudaiji Buddhist Temple, Isesaki, Japan \\ Correspondence: Koshun Suto, Chudaiji Buddhist Temple, Isesaki, Japan. Tel: 81-270-23-9980. E-mail: \\ koshun_suto129@mbr.nifty.com
}

Received: November 27, 2016

Accepted: December 16, 2016

Online Published: January 2, 2017

doi:10.5539/apr.v9n1p70

URL: http://dx.doi.org/10.5539/apr.v9n1p70

\begin{abstract}
The hydrogen atom has ultra-low energy levels. This paper calls hydrogen atoms at these energy levels "dark hydrogen atoms." A dark hydrogen atom is formed from a proton with positive mass, and an electron with negative mass. This paper claims that, if the interpretation of triplet production is changed, then the existence of electrons forming dark hydrogen has already been indirectly demonstrated. The radius of dark matter (DM), exemplified by the dark hydrogen atom, is extremely small. However, this matter can exist in a state of high density, and thus can act as a tremendous source of gravitational force, far greater than ordinary matter. This paper predicts that dark atoms, dark molecules, and various types of DM combining such particles are the true nature of the unknown mass (source of gravitational force).
\end{abstract}

Keywords: Dark matter, Electron with negative mass, Einstein's energy-momentum relationship, Hydrogen atom, Ultra-low energy levels, Triplet production

\section{Introduction}

The universe is currently thought to contain matter whose true nature is unknown, in quantities far exceeding the known forms of matter such as atoms, molecules and celestial objects. Historically, the existence of this unknown matter was first pointed out by F. Zwicky in 1933.

In the latter half of the 1970s, it was ascertained, through highly precise observations by Vera Rubin, that an unknown source of gravity is present in galaxies. Today, the existence of this unknown mass (source of gravitational force) called "dark matter" (DM) is supported by many scientists. DM is thought to have the following characteristics.

(1) It is widely present in galactic systems.

(2) It is electrically neutral.

(3) It has considerable mass.

(4) It cannot be observed optically (it does not emit light).

DM candidates can be roughly divided into two types: elementary particle candidates and astrophysical candidates. The leading elementary particle candidate is a Weakly Interacting Massive Particles (WIMPs).

In addition, a new theory was announced last year which posits another force dominating the world of the extremely small, and the existence of a Strongly Interacting Massive Particle (SIMP) (Hochberg, 2015).

The simplest model assumes one type of particle is involved in DM. However, since the 1990s various experiments have attempted to directly detect WIMPs, but no definitive signs suggesting the existence of WIMPs have been found. Therefore, some scientists have doubts about the current theory, and have begun to also consider models of DM comprised of multiple particles (Kaplan, 2010; Fan, 2013).

In 2014, the author published papers indicating the existence of hydrogen atoms at ultra-low energy levels (Suto, 2014: Suto, 2015). However, these papers have still not attracted the notice of scientists. Therefore the author decided to present those results again in this paper, while adding some new perspectives.

\section{Equation serving as the basis for deriving ultra-low energy levels of the hydrogen atom}

Letting $m_{0} c^{2}$ be the rest mass energy and $\boldsymbol{p}$ the momentum of an object or a particle existing in free space, Einstein's energy-momentum relationship is given by the following equation: 


$$
\left(m c^{2}\right)^{2}=\boldsymbol{p}^{2} c^{2}+\left(m_{0} c^{2}\right)^{2}
$$

Here, $m c^{2}$ is the relativistic energy.

In contrast, the author has derived the following relationship for the bound electron in a hydrogen atom, which must take into account the Coulomb potential (Suto, 2011).

$$
\left(m_{\mathrm{re}, n} c^{2}\right)^{2}+\boldsymbol{p}_{n}^{2} c^{2}=\left(m_{\mathrm{e}} c^{2}\right)^{2}, \quad m_{\mathrm{re}, n} c^{2}=m_{\mathrm{e}} c^{2}+E_{n} . \quad n=1,2 \cdots
$$

Here, $m_{\mathrm{re}, n} c^{2}$ is the relativistic energy of the electron, and the electron's energy is described on an absolute scale. In this case, it is natural to regard $\boldsymbol{p}$ as angular momentum.

In Relationship (2), $E_{n}$ is the total mechanical energy of a hydrogen atom derived from classical quantum theory, and it is given by the following equation:

$$
\begin{aligned}
& E_{n}=-\frac{1}{2}\left(\frac{1}{4 \pi \varepsilon_{0}}\right)^{2} \frac{m_{\mathrm{e}} e^{4}}{\hbar^{2}} \frac{1}{n^{2}} \\
& =-\frac{\alpha^{2} m_{\mathrm{e}} c^{2}}{2 n^{2}} . \quad n=1,2 \cdots .
\end{aligned}
$$

Here, $\alpha$ is the fine structure constant as follows.

$$
\alpha=\frac{e^{2}}{4 \pi \varepsilon_{0} \hbar c} .
$$

In this paper, the energy of Equation (3) is indicated $E_{\mathrm{B}, n}$. Here, the $\mathrm{B}$ in $E_{\mathrm{B}}$ signifies the energy equation derived by Bohr.

The relativistic energy of the hydrogen atom obtained from the Dirac relativistic wave equation is given by the following equation (Schiff, 1968):

$$
E=m_{\mathrm{e}} c^{2}\left[1-\frac{\alpha^{2}}{2 n^{2}}-\frac{\alpha^{4}}{2 n^{4}}\left(\frac{n}{|k|}-\frac{3}{4}\right)\right] .
$$

It is important to note that energy here is defined on an absolute scale. In this paper, the energy of Equation (5) is indicated $E_{\mathrm{D}, n}$. Here, the $\mathrm{D}$ in $E_{\mathrm{D}}$ signifies the energy equation derived by Dirac.

If we ignore the third term of this equation and define it as an approximation, Equation (5) can be written as follows.

$$
\begin{gathered}
E_{\mathrm{D}, n}=m_{\mathrm{e}} c^{2}-\frac{1}{2}\left(\frac{1}{4 \pi \varepsilon_{0}}\right)^{2} \frac{m_{\mathrm{e}} e^{4}}{\hbar^{2}} \frac{1}{n^{2}} \\
=m_{\mathrm{e}} c^{2}+E_{\mathrm{B}, n} .
\end{gathered}
$$

$E_{\mathrm{D}, n}$ of Equation (6) defines an absolute quantity, which includes the electron's rest mass energy. Whereas $E_{n}$ in Equation (3) expresses the reduction in rest mass energy of the electron, $E_{\mathrm{D}, n}$ in Equation (6) expresses the remaining amount of rest mass energy of the electron. Taking these facts into account, the relativistic energy $E_{\mathrm{re}, n}$ of the electron is defined as follows.

$$
E_{\mathrm{re}, n}=m_{\mathrm{re}, n} c^{2}=m_{\mathrm{e}} c^{2}+E_{n} . \quad n=1,2 \cdots .
$$

Here, $m_{\mathrm{re}, n}$ is the relativistic mass of the electron. From Equations (1) and (2) it is evident that, if a stationary electron begins to move in free space, or is incorporated into an atom, then the energy which serves as the departure point is the rest mass energy.

Incidentally, according to the virial theorem, the following relation holds between $K$ and $V$ : 


$$
\langle K\rangle=-\frac{1}{2}\langle V\rangle .
$$

Here, $K$ is the kinetic energy of the entire system, and $V$ is the potential energy of the entire system.

The average time of $K$ is equal to $-1 / 2$ the time average of $V$. Also, the sum of the time average $K$ and the time average of the total mechanical energy $E$ of the entire system becomes 0 . That is,

$$
\langle K\rangle+\langle E\rangle=0 .
$$

Next, if Equations (8) and (9) are combined, the result is as follows:

$$
\langle E\rangle=-\langle K\rangle=\frac{1}{2}\langle V\rangle .
$$

Taking these facts into account, the author presented the following equation as an equation indicating the relationship between the rest mass energy and potential energy of the electron in the electrostatic field of the proton (Suto, 2009).

$$
V(r)=-\Delta m_{\mathrm{e}} c^{2}
$$

According to this equation, the potential energy corresponds to the reduction in rest mass energy of the electron in the atom. Here, if Relationship (2) is rewritten taking into account Equation (10), the following two equations are obtained.

$$
\begin{gathered}
\left(m_{\mathrm{e}} c^{2}-\frac{\alpha^{2} m_{\mathrm{e}} c^{2}}{2 n^{2}}\right)^{2}+\boldsymbol{p}_{n}^{2} c^{2}=\left(m_{\mathrm{e}} c^{2}\right)^{2} . \\
{\left[m_{\mathrm{e}} c^{2}+\frac{V(x)}{2}\right]^{2}+\boldsymbol{p}_{n}^{2} c^{2}=\left(m_{\mathrm{e}} c^{2}\right)^{2} .}
\end{gathered}
$$

The following $p_{n}^{2}$ can be found from Equation (12).

$$
p_{n}^{2}=\left(m_{\mathrm{e}} c\right)^{2} \frac{\alpha^{2}}{n^{2}}\left(1-\frac{\alpha^{2}}{4 n^{2}}\right)
$$

Also, the potential energy in the hydrogen atom can be expressed with the following equation:

$$
V_{n}=-\frac{1}{4 \pi \varepsilon_{0}} \frac{e^{2}}{r_{n}}=-\frac{\alpha \hbar c}{r_{n}} .
$$

Now, if this value is substituted into Equation (13), the result is as follows:

$$
\left(m_{\mathrm{e}} c^{2}-\frac{\alpha \hbar c}{2 r_{n}}\right)^{2}+\boldsymbol{p}_{n}^{2} c^{2}=\left(m_{\mathrm{e}} c^{2}\right)^{2} .
$$

Expanding and rearranging this equation, it is possible to obtain the following quadratic equation for $r_{n}$ :

$$
p_{n}^{2} r_{n}^{2}-\alpha \hbar m_{\mathrm{e}} c r_{n}+\frac{\alpha^{2} \hbar^{2}}{4}=0
$$

If the value of Equation (14) is substituted into Equation (17), the following solution is obtained:

$$
r_{n}=\frac{\alpha \hbar m_{\mathrm{e}} c \pm\left(\alpha^{2} \hbar^{2} m_{\mathrm{e}}^{2} c^{2}-p_{n}^{2} \alpha^{2} \hbar^{2}\right)^{1 / 2}}{2 p_{n}^{2}}
$$




$$
=\left[r_{\mathrm{e}} \pm r_{\mathrm{e}}\left(1-\frac{\alpha^{2}}{2 n^{2}}\right)\right] \frac{n^{2}}{2 \alpha^{2}}\left(1-\frac{\alpha^{2}}{4 n^{2}}\right)^{-1} .
$$

Here, $r_{\mathrm{e}}$ is the classical electron radius as following:

$$
r_{\mathrm{e}}=\frac{1}{4 \pi \varepsilon_{0}} \frac{e^{2}}{m_{\mathrm{e}} c^{2}} .
$$

Here if $r_{n}^{+}$is taken to be the larger of the two solutions obtained from Equation (18), and $r_{n}^{-}$the smaller, then $r_{n}^{+}$ and $r_{n}^{-}$are as follows:

$$
\begin{gathered}
r_{n}^{+}=\left(\frac{n}{\alpha}\right)^{2} r_{\mathrm{e}}=n^{2} a_{\mathrm{B}} . \\
r_{n}^{-}=\frac{r_{\mathrm{e}}}{4}\left(1-\frac{\alpha^{2}}{4 n^{2}}\right)^{-1}, \quad \frac{r_{\mathrm{e}}}{4}<r_{n}^{-} .
\end{gathered}
$$

In Equation (21) the radius approaches $r_{\mathrm{e}} / 4$ with $n \rightarrow \infty$. Therefore this paper predicts that $r_{\mathrm{e}} / 4$ will be the radius of the atomic nucleus (the proton).

These values of $r_{n}^{+}$and $r_{n}^{-}$are substituted into the following equation.

$$
E_{n}=-\frac{1}{2} \frac{1}{4 \pi \varepsilon_{0}} \frac{e^{2}}{r_{n}} .
$$

Here, if $E_{n}^{+}$is taken to be the energy obtained by substituting $r_{n}^{+}$for $r_{n}$ in Equation (22), and similarly $E_{n}^{-}$is taken to be the energy obtained by substituting $r_{n}^{-}$for $r_{n}$, then $E_{n}^{+}$and $E_{n}^{-}$are as follows:

$$
\begin{gathered}
E_{n}^{+}=E_{\mathrm{B}, n}=-\frac{\alpha^{2} m_{\mathrm{e}} c^{2}}{2 n^{2}} . \\
E_{n}^{-}=-2 m_{\mathrm{e}} c^{2}+\frac{\alpha^{2} m_{\mathrm{e}} c^{2}}{2 n^{2}} .
\end{gathered}
$$

If these energies are described on an absolute scale using $E_{\mathrm{re}, n}$ defined in Equation (7), the values are as follows:

$$
\begin{gathered}
E_{\mathrm{re}, n}^{+}=E_{\mathrm{D}, n}=m_{\mathrm{e}} c^{2}+E_{n}^{+}=m_{\mathrm{e}} c^{2}-\frac{\alpha^{2} m_{\mathrm{e}} c^{2}}{2 n^{2}} . \\
E_{\mathrm{re}, n}^{-}=m_{\mathrm{re}, n}^{-} c^{2}=m_{\mathrm{e}} c^{2}+E_{n}^{-}=-m_{\mathrm{e}} c^{2}+\frac{\alpha^{2} m_{\mathrm{e}} c^{2}}{2 n^{2}} .
\end{gathered}
$$

If the above is indicated graphically, the result is as follows (see Fig. 1):

If the relativistic mass of the electron is taken to be $m_{\mathrm{re}, n}$, then the negative solution can be written as follows:

$$
m_{\mathrm{re}, n}^{-}=-m_{\mathrm{e}}\left(1-\frac{\alpha^{2}}{2 n^{2}}\right)<0 .
$$

From this it can be predicted that an electron at the energy levels in Equation (26) has negative mass.

In this paper, matter at this energy levels was tentatively called the "hydrogen atom at an ultra-low energy levels." However, there is a problem with that name. Chemically, this matter cannot be treated in the same group as the ordinary hydrogen atom. Therefore, in this paper it was decided to call matter at the energy levels in Equation (26) the "dark hydrogen atom" and thereby distinguish it from the ordinary hydrogen atom. 


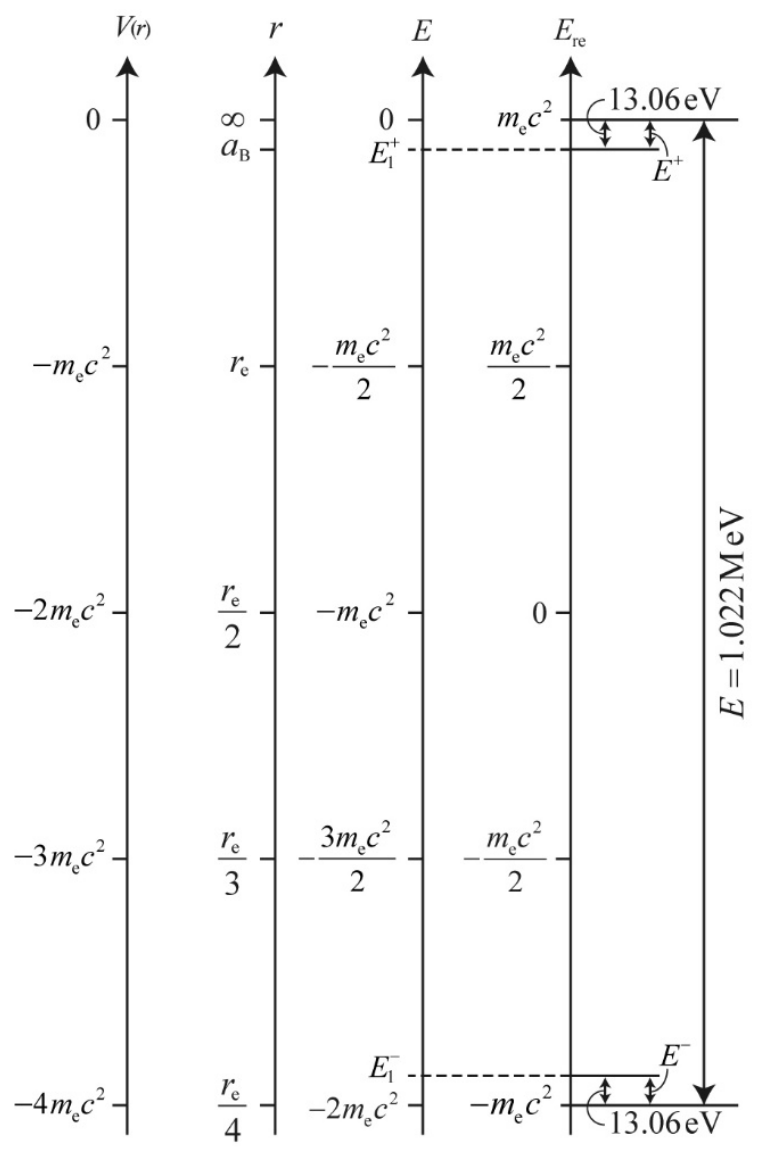

Figure 1 . The energy levels of the hydrogen atom predicted by classical quantum theory $E^{+}$and the new energy levels whose existence has been indicated by this paper $E^{-}$.

\section{Discussion}

How can hydrogen atoms in this energy state be verified? This paper looks at triplet production.

It is generally assumed that in triplet production, in which 2 electrons and 1 positron are created, electron-pair creation occurs not near the atomic nucleus, but near the electron in the outer shell orbital. A total of three particles are created in this case: one outer shell electron forming the atom, and a positron and electron created through pair production. However, in this model, $\left(1.022 \mathrm{MeV}-E_{n}^{+}\right)$should be sufficient as the necessary photon energy. If an energy of $2.044 \mathrm{MeV}\left(4 m_{\mathrm{e}} c^{2}\right)$, is needed for triplet production, then the recoiled electron should be regarded as being at an ultra-low energy level.

Now, consider the case where an incident $\gamma$-ray has the energy corresponding to the mass of 4 electrons (2.044 $\mathrm{MeV}$ ). If this is discussed classically, the $\gamma$-ray can create an electron and positron near $r=r_{\mathrm{e}} / 2$ (see Fig.2).

Even if $1.022 \mathrm{MeV}$ of energy is consumed in this pair creation, the $\gamma$-ray still has the energy of corresponding to the mass of 2 electrons $(1.022 \mathrm{MeV})$. If the $\gamma$-ray gives energy to an electron in the orbital near the proton, the electron will be excited and appear in free space. As a result, 2 electrons and 1 positron will appear in free space.

One of these two electrons had a negative mass before appearing in free space. When this electron absorbs a $\gamma$-ray, and acquires energy exceeding $m_{\mathrm{e}} c^{2}$, then the electron becomes observable in free space. If the energy of the electron is $m_{\mathrm{e}} c^{2}+E_{n}^{+}$, the electron may transition to energy levels of an ordinary hydrogen atom. This paper predicts that hydrogen atoms can be produced from dark hydrogen atoms.

However, the author is pessimistic about the reverse process. As is evident from Equations (10) and (11), common sense suggests that the minimum theoretically permissible energy of the hydrogen atom is $E=-m_{\mathrm{e}} c^{2} / 2$ (i.e., $\left.E_{\mathrm{re}}=m_{\mathrm{e}} c^{2} / 2\right)$. Based on this view, an electron forming a hydrogen atom cannot emit the photon necessary to transition to the energy levels of the dark hydrogen atom. 


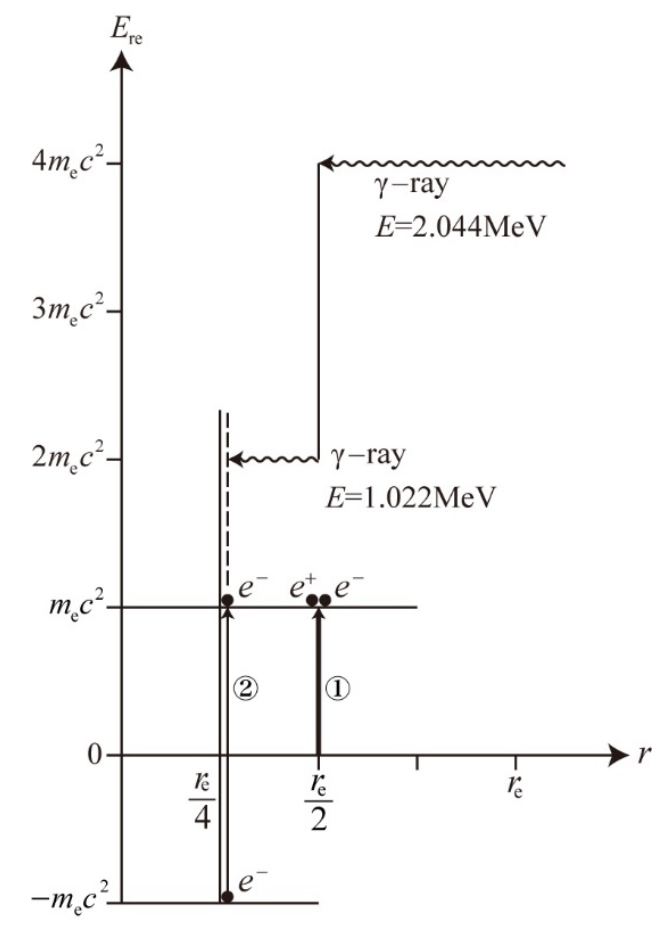

Figure 2. Interpretation of this paper regarding triplet production

This $\gamma$-ray will give $1.022 \mathrm{MeV}$ of energy to the virtual particles at $r=r_{\mathrm{e}} / 2$, and an electron-positron pair will be created ( $\uparrow$ (1). When this $\gamma$-ray approaches closer to the atomic nuclear, and the electron in the orbital around the proton absorbs this energy, the electron will be excited and appear in free space ( $\uparrow(2)$ ). Arrows (1) and (2) show the change in particle energy. The end point of an arrow does not indicate the position where the particle was produced.

This paper points out that one of the two electrons which appears is an electron in the $E_{n}^{-}$state. A hydrogen atom in the $E_{n}^{-}$state will henceforth be called a "dark hydrogen atom" in this paper, and indicated as ${ }_{\mathrm{D}} \mathrm{H}$ (where the D stands for "dark"). Also, the $E_{n}^{-}$in Equation (24) will be indicated as ${ }_{\mathrm{D}} E_{n}$, and $E_{n}^{+}$will be returned to the original symbol $E_{n}$. In addition, the $E_{\mathrm{re}, n}^{-}$in Equation (26) will be expressed $\mathrm{as}_{\mathrm{D}} E_{\mathrm{re}, n}$, and $E_{\mathrm{re}, n}^{+}$will be returned to the original symbol $E_{\mathrm{re}, n}$.

Furthermore, the hydrogen molecule produced from ${ }_{\mathrm{D}} \mathrm{H}$ will be called "dark hydrogen molecule", and indicated as ${ }_{\mathrm{D}} \mathrm{H}_{2}$.

This paper regards the existence of ${ }_{\mathrm{D}} \mathrm{H}$ as demonstrated, albeit indirectly, by the fact the electrons forming ${ }_{\mathrm{D}} \mathrm{H}$ have been observed.

\section{Conclusion}

This paper concludes that ${ }_{\mathrm{D}} \mathrm{H}$ is formed from a single proton with positive mass (energy), and a single electron with negative mass.

It can be predicted that ${ }_{\mathrm{D}} \mathrm{H}$ produces ${ }_{\mathrm{D}} \mathrm{H}_{2}$, and that other types of atoms produces dark molecules from dark atoms. Moreover, it is likely possible to produce DM from these molecules. All of these kinds of DM share the characteristics that the mass of the electrons forming the matter is always negative.

The radius of DM, exemplified by the dark hydrogen atom, is extremely small. However, this matter can exist in a state of high density, and thus can act as a tremendous source of gravitational force, far greater than ordinary matter. This paper predicts that dark atoms, dark molecules, and various types of DM combining such particles are the true nature of the unknown mass (energy).

Here, if $E_{n}^{-}$in Equation (24) is expressed as ${ }_{\mathrm{D}} E_{n}$, and $E_{\mathrm{re}, n}^{-}$in Equation (26) is expressed as ${ }_{\mathrm{D}} E_{\mathrm{re}, n}$, then the energy levels described by the levels of classical quantum theory can be expressed as follows (here, the $\mathrm{D}$ in ${ }_{\mathrm{D}} E_{n}$ signifies the energy of $\left.{ }_{D} H\right)$. 


$$
\begin{gathered}
{ }_{\mathrm{D}} E_{n}=-2 m_{\mathrm{e}} c^{2}+\frac{\alpha^{2} m_{\mathrm{e}} c^{2}}{2 n^{2}}, \quad n=1,2 \cdots \\
{ }_{\mathrm{D}} E_{\mathrm{re}, n}={ }_{\mathrm{D}} m_{\mathrm{re}, n} c^{2}=-m_{\mathrm{e}} c^{2}+\frac{\alpha^{2} m_{\mathrm{e}} c^{2}}{2 n^{2}}, \quad n=1,2 \cdots
\end{gathered}
$$

Also, if $m_{\mathrm{re}, n}^{-}$in Equation (27) is expressed as ${ }_{\mathrm{D}} m_{\mathrm{re}, n}$, then the relativistic mass of dark electron can be expressed as follows.

$$
{ }_{\mathrm{D}} m_{\mathrm{re}, n}=-m_{\mathrm{e}}\left(1-\frac{\alpha^{2}}{2 n^{2}}\right)<0 .
$$

This paper predicts that aggregates of DM form a dark universe separate from our universe made up of known matter.

\section{Acknowledgments}

I would like to express my thanks to the staff at ACN Translation Services for their translation assistance. Also, I wish to express my gratitude to Mr. H. Shimada for drawing figures.

\section{References}

Hochberg, Y., Kuflik, E., Murayama, H., Volansky, T., \& Wacker, J. G. (2015). Model for thermal relic dark matter of strongly interacting massive particles. Physical review letters, 115(2), 021301. https://doi.org/10.1103/PhysRevLett.115.021301

Kaplan, D. E., Krnjaic, G. Z., Rehermann, K. R., \& Wells, C. M. (2010). Atomic dark matter. Journal of Cosmology and Astroparticle Physics, 2010(05), 021.http://dx.doi.org/10.1088/1475-7516/2010/05/021

Fan, J., Katz, A., Randall, L., \& Reece, M. (2013). Dark-disk universe. Physical review letters, 110(21), 211302. https://doi.org/10.1103/PhysRevLett.110.211302

Suto, K. (2009). True nature of potential energy of a hydrogen atom. Physics Essays, 22(2), 135-139. http://dx.doi.org/10.4006/1.3092779

Suto, K. (2011). An energy-momentum relationship for a bound electron inside a hydrogen atom. Physics Essays, 24(2), 301-307. http://dx.doi.org/10.4006/1.3583810

Suto, K. (2014). Previously unknown ultra-low energy level of the hydrogen atom whose existence can be predicted. Applied Physics Research, 6(6), 64-73. http://dx.doi.org/10.5539/apr.v6n6p64

Suto, K. (2015). Presentation of strong candidates for dark matter. Global Journal of science frontier research: A, 15(7), 1.0, 1-6. https://doi.org/10.17406/GJSFRFVOL15IS7PG1

Schiff, L. I. (1968) Quantum Mechanics (p. 472). New York: McGraw-Hill.

\section{Copyrights}

Copyright for this article is retained by the author(s), with first publication rights granted to the journal.

This is an open-access article distributed under the terms and conditions of the Creative Commons Attribution license (http://creativecommons.org/licenses/by/4.0/). 